\title{
Angiomatous Nasal Polyp: A Condition Difficult to Diagnose
}

\author{
${ }^{1}$ Neeti Verma, ${ }^{2}$ Narender Kumar, ${ }^{3}$ Ramesh Azad, ${ }^{4}$ Neelam Sharma \\ ${ }^{1}$ Registrar, Department of ENT, Indra Gandhi Medical College, Shimla, Himachal Pradesh, India \\ ${ }^{2}$ Professor and Head, Department of ENT, Indra Gandhi Medical College, Shimla, Himachal Pradesh, India \\ ${ }^{3}$ Associate Professor, Department of ENT, Indra Gandhi Medical College, Shimla, Himachal Pradesh, India \\ ${ }^{4}$ Associate Professor, Department of Pathology, Indra Gandhi Medical College, Shimla, Himachal Pradesh, India
}

Correspondence: Neeti Verma, Registrar, Department of ENT, Indra Gandhi Medical College, Shimla, Himachal Pradesh, India

\section{ABSTRACT}

Introduction: Angiectatic nasal polyp (ANP) is a rare entity of inflammatory sinonasal polyp, constituting 4 to $5 \%$ of all nasal polyps. ${ }^{1}$ An angiomatous polyp originates from a sinochoanal polyp, either an antrochoanal or a sphenochoanal polyp. Their hallmark feature is extensive vascular proliferation and ectasia with deposition of pseudoamyloid and the presence of atypical stromal cells. This pseudoneoplastic entity is the result of extensive reactive and reparative changes in inflammatory SNPS.

Case report: We came across an interesting case of angiomatous nasal polyp. CT finding also supported the clinical impression of neoplastic mass but repeated biopsies were negative for malignancy. The mass was excised by lateral rhinotomy and the final impression of angiectatic nasal polyp was made.

Discussion and conclusion: Only few studies have been done on this topic and literature is also scant. Thus, awareness of their existence is of considerable importance. Differential diagnosis includes angiofibroma, malignancy, inverted papillomas and hemangiomas.

Keywords: Antrochoanal polyp, Angiomatous polyp, Necrosis, Sphenochoanal polyp.

\section{INTRODUCTION}

Nasal polyps are chronic edematous mucosa attached by a pedicle, arising from nose or paranasal sinus, having a characteristic appearance and a common occurrence. ${ }^{2}$ On gross examination, the sinonasal polyps appear as gelatinous, semitransparent masses and microscopically, they show edematous lamina propria with infiltrates of inflammatory cells, eosinophils being in majority. ${ }^{3} \mathrm{~A}$ vascular permeability factor produced by plasma cells leads to the interstitial edema. V ascular congestion along with obstruction of the outflow of tissue fluids are other factors contributing. Contrary to angiomatous polyp, the inflammatory SN Ps have a decreased blood flow and a lesser number of blood vessels compared with normal nasal mucosa. ${ }^{3}$ Based on predominant infiltrate seen on histological evaluation, inflammatory SNPs have been classified into five types: Edematous, glandular, fibrous, cystic and angiectatic or angiomatous (rare representing only $4-5 \%)^{2}$

\section{CASE REPORT}

A 32-year-old female from $\mathrm{Nepal}$, presented in our department in A ugust 2009, with nasal obstruction, mass and occasional bleeding from left side of nose for four months. It was associated with headache, inability to smell, swelling over the nose, left cheek and medial canthus of eye with protrusion and excessive watering from left eye for three months. There was no history of excessive sneezing, watery nasal discharge. No history of TB, asthma, atopy in family. Past and personal history was insignificant and general physical examination did not reveal anything contributory.

On local examination (Figs 1 and 2), there was a visible swelling over dorsum of the nose and medial canthus of the left eye. There was proptosis along with lateral displacement of eyeball, ocular movements were restricted but the visual acquity was $6 / 6$ in right eye and $6 / 60$ in the left eye.

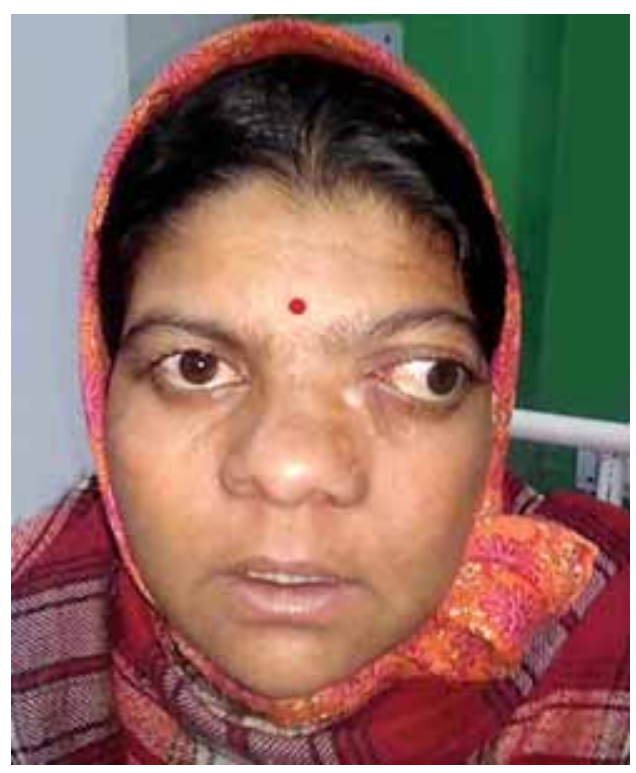

Fig. 1: Preoperative photograph of the patient (frontal view) 


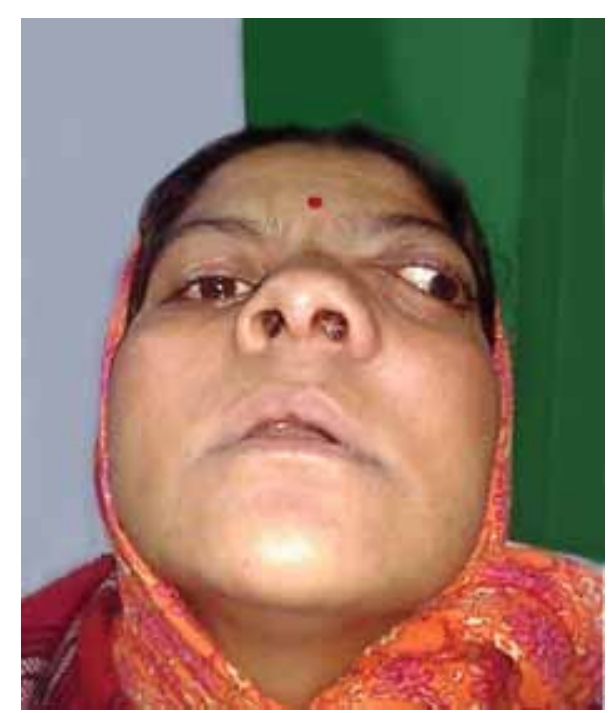

Fig. 2: Preoperative photograph of the patient (basal view)

Tenderness could be appreciated over the dorsum of nose, medial canthus of eye and maxillary sinus. Inferior orbital margins were palpable along the whole length. Anterior hygrometery was decreased on the left side. On anterior rhinoscopy, there was discharge and a reddish polypoidal mass was visible, which was less sensitive to touch, did not bleed on touch, could be probed medially but not laterally. The septum was pushed to the right side.

The routine investigations were normal. X-ray PNS showed opacity in left maxillary antrum and left nasal cavity. B oth $\mathrm{KOH}$ mount and fungal cultures were negative. Punch biopsy was sent thrice, but showed only polypoidal mucosa with inflammatory infiltrate, with no evidence of malignancy. HRCT nose and PNS (Fig. 3) showed a homogeneous density mass filling the whole of left maxillary antrum, superiorly eroding the medial wall of orbit, though orbital contents appeared to be uninvolved. On CT, the first possibility of a fungal mass was kept and second of a tumor of maxillary sinus.

Based on these findings, a provisional diagnosis of growth of maxillary sinus and nasal cavity on left side was made and patient was taken up for surgery by lateral rhinotomy approach. Medial maxillectomy with frontoethmoidectomy was performed on the left side. A nterior wall of maxilla was dehiscent, its medial wall and lamina papyracea were destroyed, a large gelatinous mass was filling the whole of the maxillary antrum and septum was thinned out and was pushed to right. It was also involving the ethmoids, frontal and sphenoid sinuses.

Histopathological examination (Figs 4 and 5) showed that the epithelium was ciliated pseudostratified columnar, there was loose myxoid stroma, mucous glands, lymphocytes, plasma cells, neutrophils and eosinophils. Few sections showed large dilated thick and thin walled blood vessels, extracellular eosinophilic material and fragments

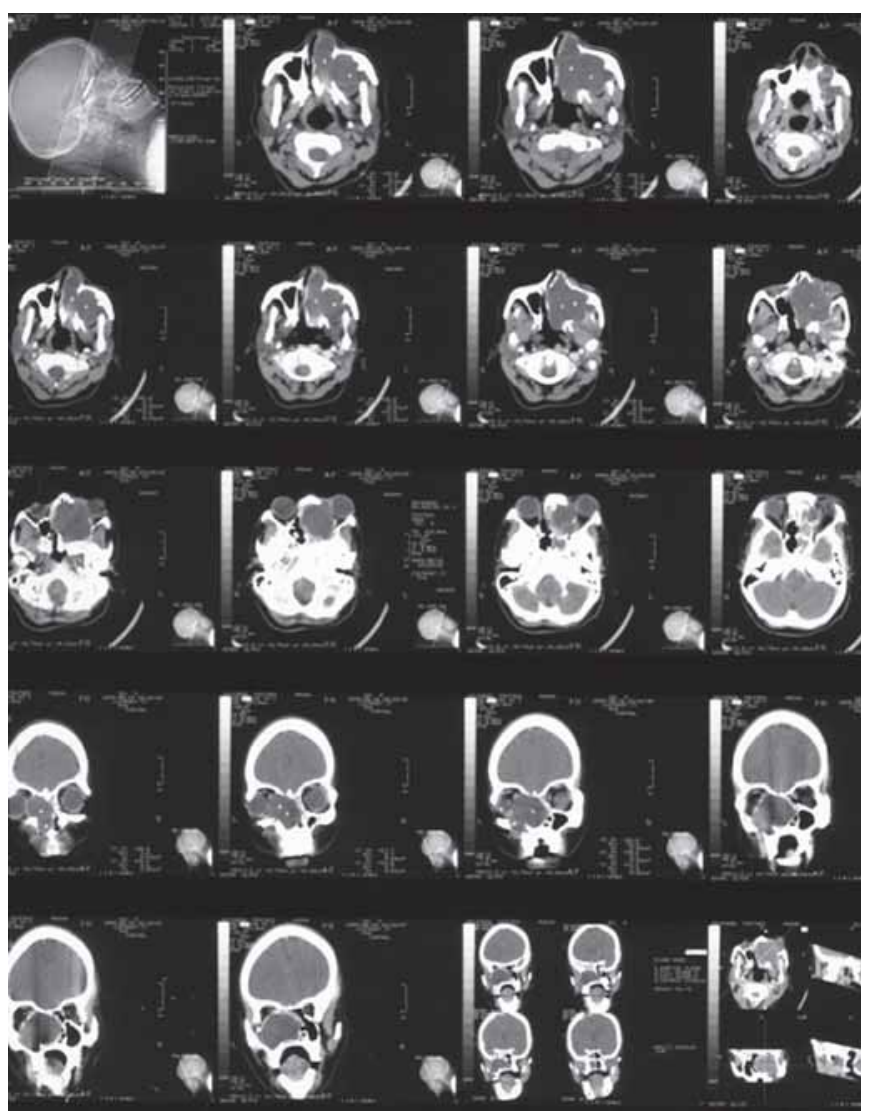

Fig. 3: HRCT nose and PNS showed a homogeneous density mass filling the whole of left maxillary antrum and superiorly eroding the medial wall of orbit. The orbital contents appear to be uninvolved

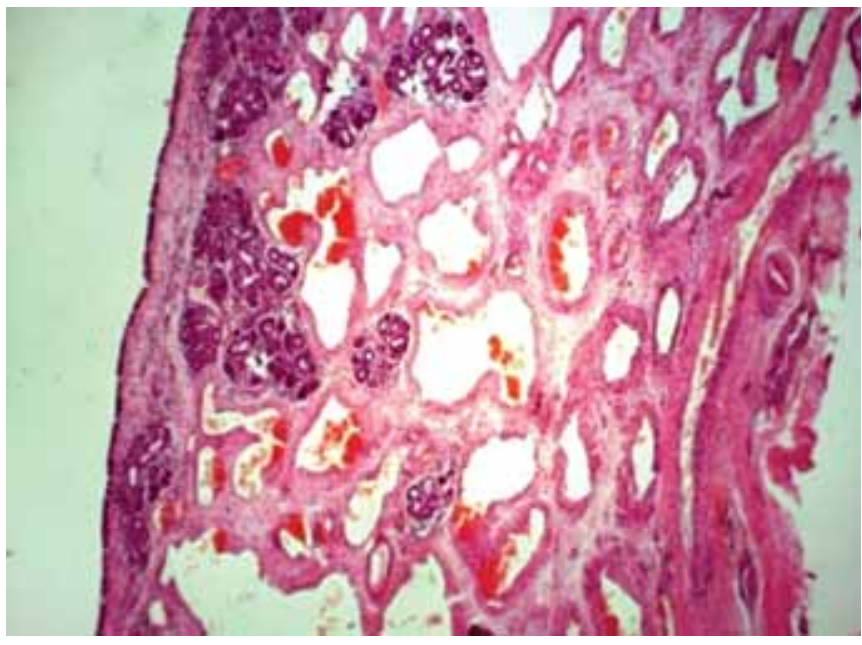

Fig. 4: Histopathology $(H \& E \times 10)$ showing dilated thin walled blood vessels and extracellular eosinophilic material

of necrotic bone. A final impression of angiomatous/ angioectatic polyp was made.

\section{DISCUSSION}

A ngiomatous polyps are fibrosed, vascularized nasal polyp, probably a response to minor trauma. ${ }^{4}$ Choanal polyps may occasionally degenerate into angiomatous polyps. ${ }^{5}$ The process of degeneration begins with compression and occlusion of vessels at vulnerable sites in the polyp, that is 


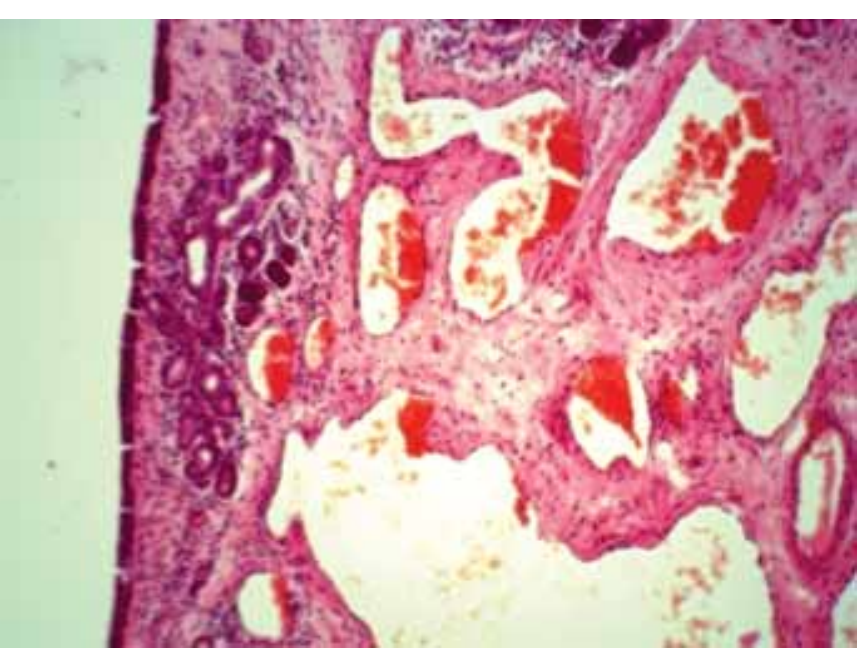

Fig. 5: Histopathology $(H \& E \times 40)$ showing thin dilated blood vessels and extracellular eosinophilic material

the ostial opening, and the dependent areas of the polyp. ${ }^{5,6}$ This leads to stasis of blood, followed by thinning and dilatation of blood vessel sal ong with thrombosis and infarct. ${ }^{7}$ A blood component like fibrin and platel ets extravasates through these thinned capillary-like vessels. Grossly, they havean unusual inhomogeneous appearanceand texture and a foul smell. ${ }^{8} \mathrm{M}$ icroscopically, the A N Ps are characterized by large numbers of dilated capillary spaces, scanty inflammatory infiltrates (mainly the plasma cells), ${ }^{8}$ and abundantamorphous, amyloid-like (butcongo red-negative) material. A ngiomatous polyps have been accompanied by the presence of pseudosarcomatous cell changes and stromal atypia (large pleomorphic spindle cells).

M ost common clinical presentation of ANPs is nasal obstruction. Other symptoms are loss of smell sensation, epistaxis, ${ }^{8}$ exophthalmoses, proptosis and visual disturbances. ${ }^{3}$ On anterior rhinoscopy, they appear as a smooth, bluish gray/reddish polypoidal mass with shiny surface. ANPs may cause extensive bone erosion and remodeling. This erosive feature raises the suspicion of a malignant process. Being vascular, they may also be confused with other benign vascular lesions, such as nasopharyngeal angiofibromas. Even on $\mathrm{CT}$, the differentiation between the two is tough. The unenhanced M R images depict the typical appearance of a sinochoanal polyp, hypointense on T1-weighted and hyperintense on T2weighted images. O $n$ gadolinium-enhanced M R images, the sinus part shows little or no peripheral enhancement; however, the nasochoanal part, corresponding to the angiomatous polyp, shows a strong enhancement. A $n$ angiomatous polyp mimics a hypervascular mass lesion on enhanced MR studies. ${ }^{9}$

The A N Ps though rare can pose a difficult diagnostic problem. These are benign, pseudoneoplastic lesions, managed on the same lines as the choanal polyps. Due to the presence of pseudosarcomatous changes, they need to be differentiated from a soft tissue sarcoma (e.g. a malignant fibrous histiocytoma).

The AN Ps should also be differentiated from capillary or cavernous hemangiomas (commonest nonepithelial tumor of nose and nasopharynx). A ngiomas are composed of irregular vascular channels lined by flattened endothelial cells embedded in edematous stroma. They are usually found in the anterior nasal septum, the turbinates and the vestibule. ${ }^{3}$

The AN Ps can be confused with juvenile nasopharyngeal angi ofibromas. ${ }^{10}$ The A NP is located primarily in the nasal fossa and not in the nasopharynx, it does not extend into the pterygopalatine fossa, the sphenoid sinus and intracranially. On angiography, ANPs have only a few demonstrable feeding vessels compared with the rich vascular supply of angiofibromas. On CT, ANPS do not enhance as well as the angiofibromas do, vascular flow voids, as seen in angiofibroma are usually not seen on M R I imaging, in case of polyps. For A NPS, excision is curative and recurrences are rare. This is in contrast to angi ofibromas, which are frank neoplasm that require either surgical excision and/or radiotherapy to prevent recurrence. The possibility of A N Ps should al ways be considered before the diagnosis of nasopharyngeal angiofibromas is considered in elderly or female patients.

A nother differential diagnosis is squamous cell carcinoma. ${ }^{3}$ Simxilarity being the aggressive clinical behavior and pseudoepitheliomatous hyperplasia. B ut during surgery, it becomes evident that the AN Ps are benign and easily excisable. A Iso, bleeding is minimal both during and after surgery.

\section{CONCLUSION}

Clinically, this rare entity closely simulates hemangiomas, angiofibromas, inverted papilloma and epithelial and mesenchymal tumors. Differentiation is extremely important as their lines of management are entirely different. Extensive vascular proliferation, accumulation of extracellular amorphous eosinophilic material and atypical stromal cells are characteristics of ANPS. Prognosis is good and recurrence is rare.

\section{REFERENCES}

1. Jumpei N ota, Y asuyuki Hinohira, Hirotaka Takahashi, Futoshi W atanabe, K iyofumi Gyo. A ngiectatic nasal polyp localized in the maxillary sinus. Japanese J of R hinology 2008;47(2): 105-08.

2. Sinha SN . Observations on histology of nasal polyps. Indian J of Otolaryngol and Head and N eck Surg M ay 2007;19(4):164-68.

3. Y fantis HG, D rachenberg CB, Gray W, Papdimitriou JC. Angiectatic nasal polyps that clinically simulate a malignant process: R eport of two cases and review of literature. A rchives of Pathol Lab M ed Mar 2000;124(3):406-10. 
4. Batsakis J, et al. Tumors of head and neck: Clinical and pathological considerations (2nd ed). B altimore: Williams and Wilkins 1979;139-43.

5. Park CS, N oh H, B ae SC, Park Y J. A ntrochoanal polyp's variant, the angiomatous nasal polyp: A case report. Korean J Otolaryngol Head and Neck Surg A pr 2006;49(4):443-46.

6. Sheahan P, Crotty PL, Hamilton S, Colreavy M, M cShane D. Infarcted angiomatous nasal polyps. Eur A rch Otolaryngol 2005;262:225-30.
7. Batsakis J G, Sneige N. Choanal and angiomatous polyps of sinonasal tract. A nn Otol Rhinol Laryngol 1992;101:623-25.

8. Sayed RH,A bu-Dief EE. D oes antrochoanal polyp presents with epistaxis? B-ENT 2007;3(3):145-47.

9. De V uysere S, Hermans R, M archal G. Sinonasal polyp and its variant-the angiomatous polyp: M RI findings. Eur Radiol 2001;11(1):55-58.

10. Som P, Cohen B, Sacher M. The angiomatous polyp and the angiofibroma: Two different lesions. Radiology 1982;144: 329-34.

\title{
Editorial Inputs
}

\section{Angiomatous Nasal Polyp: A Condition Difficult to Diagnose}

\author{
${ }^{1}$ Bachi T Hathiram, ${ }^{2}$ Vicky S Khattar \\ ${ }^{1}$ Professor and Head, Department of ENT and Head and Neck Surgery, Topiwala National Medical College and BYL Nair Charitable Hospital \\ Mumbai, Maharashtra, India \\ ${ }^{2}$ Assistant Professor, Department of ENT and Head and Neck Surgery, Topiwala National Medical College and BYL Nair Charitable Hospital \\ Mumbai, Maharashtra, India
}

Truly a diagnostic dilemma, especially preoperatively, since most of the investigations do not specifically diagnose this condition. Often there is no history of bleeding. Clinical presentation may vary from a simple antrochoanal polyp, through angiofibromas, inverted papillomas, fungal infections and malignancy.

Two of our cases had definite vascular pedicles. The first could well be seen within the maxilla after removal, with the arterial spurter, which was easily cauterised (Fig. 1).

A nother of our cases was diagnosed preoperatively as a nasopharyngeal angiofibroma (Figs 2 and 3 ). It was

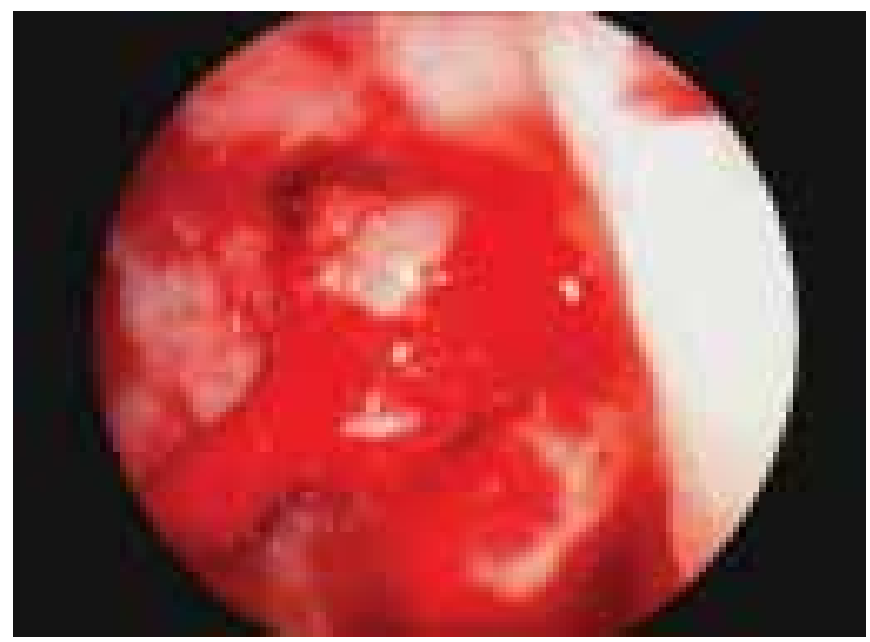

Fig. 1: Intraoperative endoscopic image showing the vascular pedicle within the left maxillary sinus, arising from the anterolateral wall, with the arterial spurter subsequently embolized with polyvinyl alcohol particles by the interventional radiology colleagues. To our surprise, the tumor has shrunk my more than $70 \%$ in size within 48 hours of embolization, the mass was excised endoscopically, and the histopathology reveal ed it to be an angiomatous polyp.

De V uysere et al $(2001)^{1}$ state that angiomatous nasal polyps correspond to the vascular compromised nasochoanal part of a sinochoanal polyp. The M RI findings of one such patient of ours diagnosed retrospectively after surgery showed typical features of a sinochoanal polyp (Figs 5 and 6), namely hypointense on T1 and hyperintense on T2 weighted images.

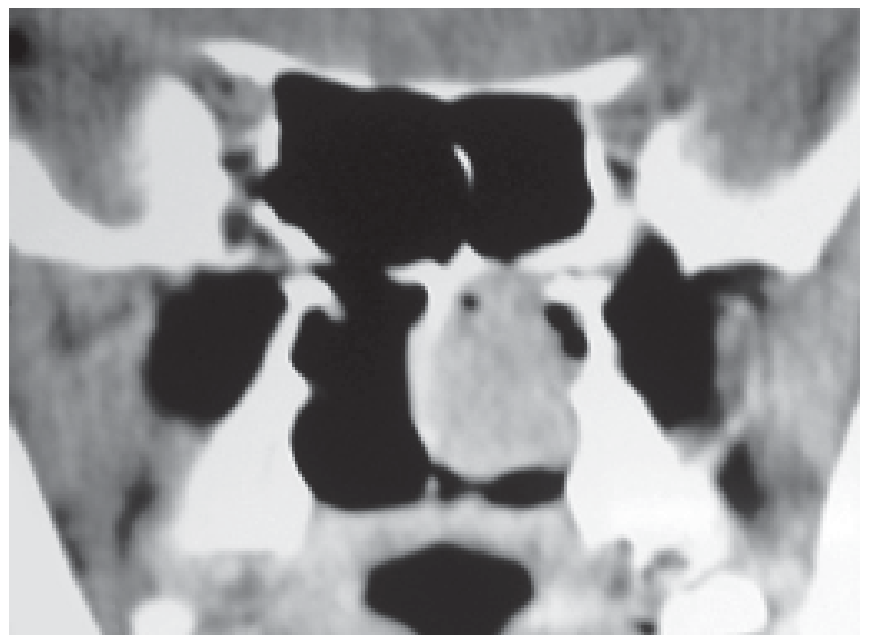

Fig. 2: Preoperative contrast enhanced coronal CT scan of the paranasal sinuses showing the enhancing mass in the left nasal cavity 


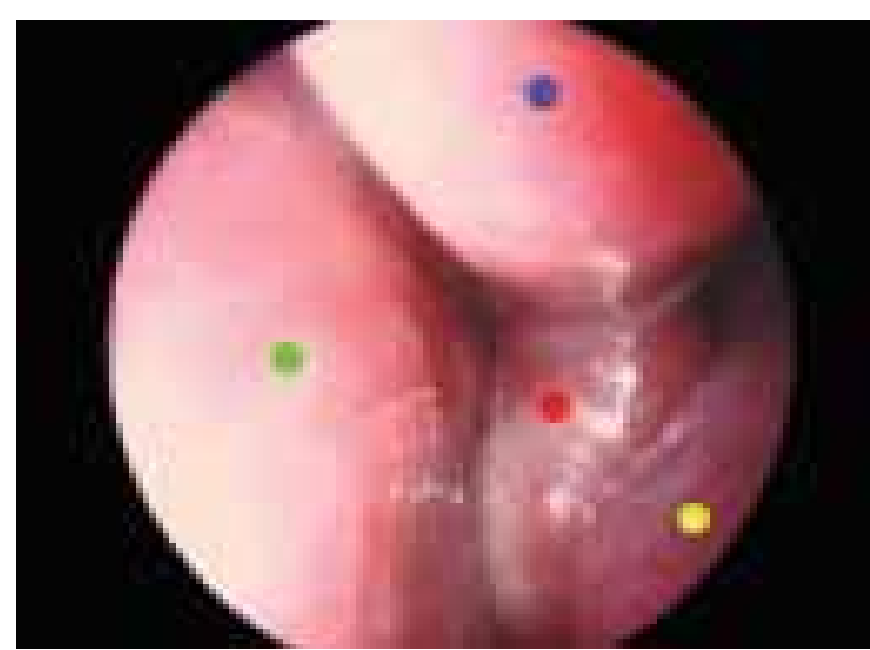

Fig. 3: Preembolization endoscopic image showing the angiomatous polyp filling the posterior half of the left nasal cavity (red dot). The septum (green dot), inferior turbinate (yellow dot) and middle turbinate (blue dot) are also seen

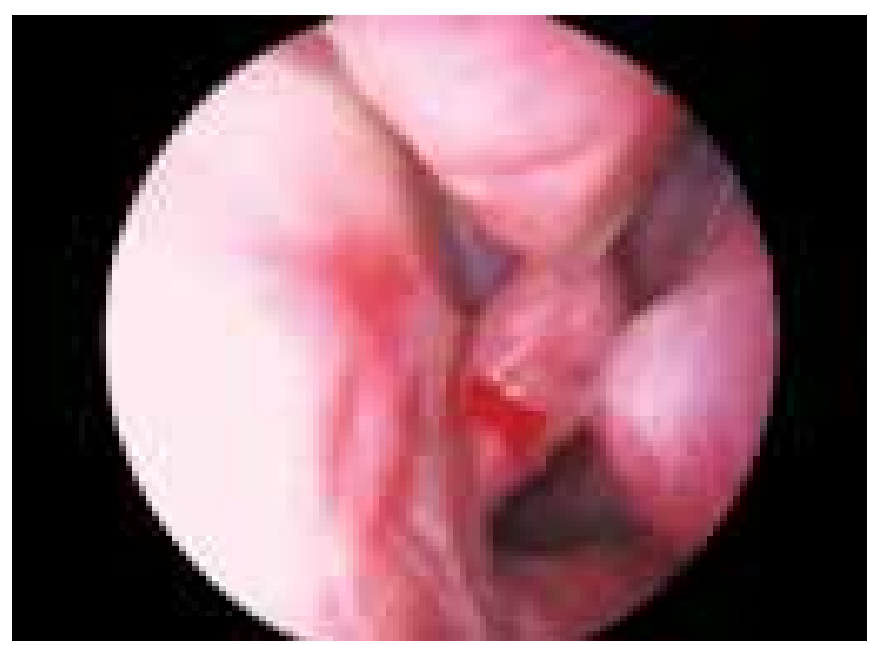

Fig. 4: Postembolization preoperative endoscopic image showing the polyp reduced by more than $70 \%$ of its volume. The origin could now be appreciated from the nasal septum

The clincher would however be the postcontrast study which would reveal the sinusal component with little or no peripheral enhancement, and the nasochoanal component to show strong enhancement, corresponding to the angiomatous component. Since our patient was diagnosed retrospectively, we could not have the contrast-enhanced M RI images.

Thetheory putforward for such masses is that thevascular compromise causes a neovascularization of the 'compromised' component, which isusually thenasochoanal part. In our first case, however, we found the 'feeder' vessel

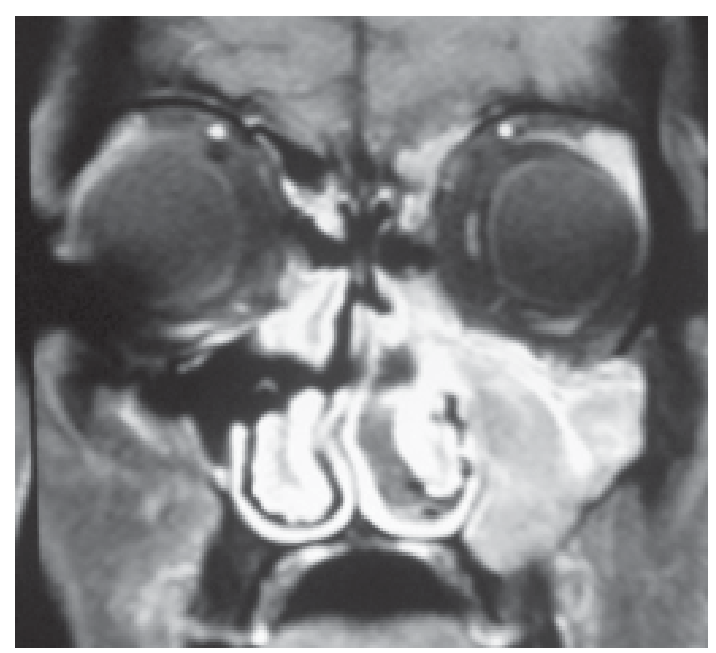

Fig. 5: T1-weighted image

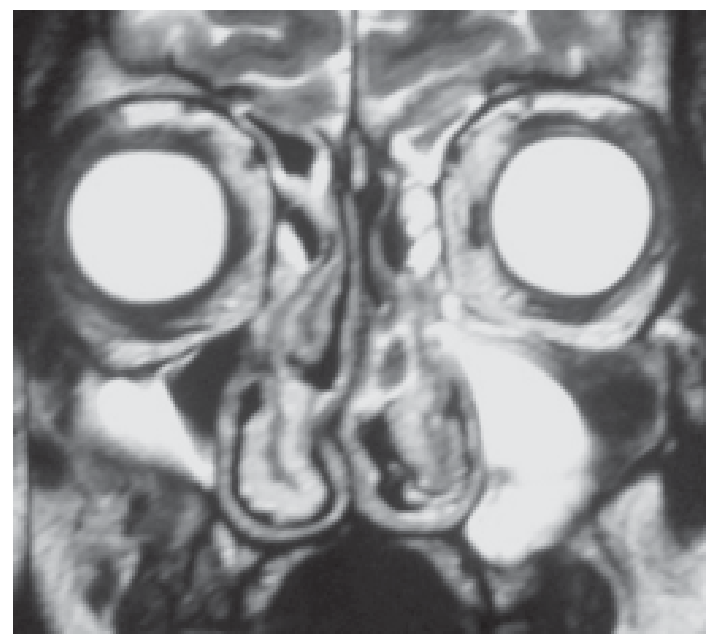

Fig. 6: T2-weighted image

to be in the lateral wall of themaxillary sinus, and the second case, the mass was found pedicled to the nasal septum. It was only the third case which ahd the typical findings of a 'angiomatous polyp.' Sincetheliterature on this entity is rare, itwould bea good idea to comparethehistology and radiology of all reported cases, to clearly define this entity.

\section{REFERENCE}

1. De V uysere S, Hermans R, M archal G. Sinochoanal polyp and its variant, the angiomatous polyp: M RI findings. Eur Radiol 2001;11(1):55-58. 